\title{
Bei asymptomatischen Karotisstenosen operieren oder stenten?
}

Fragestellung: Besteht im Langzeiterfolg und bei den Komplikationen ein Unterschied zwischen der Karotisoperation und dem Karotisstenting bei Patienten mit hochgradigen asymptomatischen Karotisstenosen?

Hintergrund: Eine Karotisstenose wird dann als asymptomatisch angesehen, wenn es im entsprechenden Gefäßgebiet weder zu einem ischämischen Insult noch zu einer transienten ischämischen Attacke (TIA) gekommen ist. Zwei große randomisierte Studien hatten vor längerer Zeit die Wirksamkeit der Karotisoperation bei asymptomatischen Karotisstenosen belegt, wenn die Komplikationsrate des Eingriffs weniger als $3 \%$ betrug. In der Folgezeit wurden dann eine ganze Reihe von offenen und später auch randomisierten Studien durchgeführt, welche die Karotisoperation mit dem Stenting bei asymptomatischen Karotisstenosen verglichen. Im vorliegenden Fall führten die Autoren eine systematische Literaturrecherche und eine Metaanalyse durch.

Moresoli P, Habib B, Reynier $P$ et al. Carotid stenting versus endarterectomy for asymptomatic carotid artery stenosis: A systematic review and meta-analysis. Stroke 2017; 48: $2150-7$
Patienten und Methodik: Die Autoren identifizierten fünf randomisierte Studien mit 3.019 Patienten mit asymptomatischen Carotisstenosen. Die CREST-Studie schloss 1.181 Patienten ein und an der ACT-I-Studie nahmen 1.453 Patienten teil. Die drei übrigen Studien hatten jeweils nur geringe Patientenzahlen. Die Beobachtungszeit nach dem Eingriff erfolgte über einen Zeitraum von drei bis zehn Jahren. Die vordefinierten Endpunkte der Metaanalyse waren periprozedurale Schlaganfälle, definiert als alle Schlaganfälle, die innerhalb von 30 Tagen nach dem Eingriff auftraten, periprozedurale nicht behindernde Schlaganfälle und periprozedurale Schlaganfälle oder Tod.

Ergebnisse: Für periprozedurale Schlaganfälle oder Todesfälle traten diese Ereignisse bei 46 von 1.747 Patienten auf, die einen Stent erhielten, und bei 14 von 1.102 Patienten, die operiert wurden. Dies entspricht einem relativen Risiko von 1,72 mit einem 95\%-Konfidenzintervall (KI) von 0,95-3,11 zu Ungunsten des Stentings. Periprozeduale Schlaganfälle mit bleibender Behinderung traten achtmal in der Stenting- und dreimal in der Operationsgruppe auf. Dies entspricht einem relativen Risiko von 1,41 mit einem $95 \%$-KI von 0,41-4,84. Periprozedurale Herzinfarkte traten zwölfmal in der Stenting- und 16-mal in der Operationsgruppe auf. Dies entspricht einem relativen Risiko von 0,55 mit einem $95 \%$-KI von $0,26-1,16$.

Schlussfolgerungen: Diese Metaanalyse zeigte, dass bei Patienten mit asymptomatischen Karotisstenosen die Komplikationsrate bei der Karotisendarteriektomie tendenziell niedriger ist als beim Karotis-Stenting.

\section{- Kommentar von Hans Christoph Diener, Essen}

\section{Warum nicht konservativ behandeln?}

Die hier vorliegende Metaanalyse von zwei großen und drei kleinen randomisierten Studien belegt ähnlich wie bei symptomatischen Karotisstenosen, dass sehr wahrscheinlich die Karotisoperation eine etwas geringere Komplikationsrate hat als das Karotis-Stenting. Dies gilt insbesondere für ältere Patienten. Die Metaanalyse adressiert allerdings eine extrem wichtig klinische Frage nicht, nämlich die, ob ein interventio-

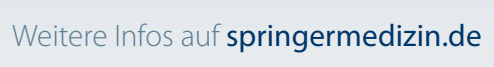

Bildgebende Darstellung hirnversorgender Gefäße

Dieser Artikel fasst die Indikationen und Methoden zur bildgebenden Darstellung hirnversorgender Gefäße zusammen. Sie finden ihn, indem Sie den Titel in die Suche eingeben. neller Eingriff bei Patienten mit asymptomatischen Karotisstenosen überhaupt notwendig ist. Die randomisierten Studien zum Stenting von intrakraniellen Stenosen haben in der SAMMPRIS-Studie gezeigt, dass eine aggressive konservative Therapie einer interventionellen Behandlung überlegen ist. Angesichts der extrem geringen Raten an Schlaganfällen bei Patienten mit asymptomatischen Karotisstenosen unter moderner medikamentöser Behandlung der Risikofaktoren ist wahrscheinlich eine konservative Therapie mindestens genauso wirksam wie ein interventioneller Eingriff. Dies wird im Moment in einer randomisierten Studie in den USA untersucht (CREST-II). Die deutsche SPACE-2-Studie, welche die Intervention mit optimaler konservativer Therapie verglich, musste leider mangels Rekrutierung vorzeitig beendet werden. Darüber hinaus muss berücksichtigt werden, dass in Deutschland weiterhin bei 70.000 Patienten pro Jahr eine asymptomatische Karotisstenose operiert oder gestentet wird, obwohl die Leitlinien der Deutschen Gesellschaft für Neurologie (DGN) hier zur Zurückhaltung aufrufen. 